\title{
Equal Opportunity and the Education System in Pakistan
}

\section{Rukhsana Zia*}

\section{Introduction}

The educational system in Pakistan is beset with multiple dilemmas and constraints. Some are underlying and deep-rooted, while others are rooted in immediate, superficial reasons. Understandably all are inter linked and each affects the other. One that pervades as an under current is the issue of lack of Equal Opportunity (EO) which takes myriad forms when translated into practice. This paper delineates the various forms the absence or imbalanced prevalence of EO takes within the context of the education system of the country, and primary education in particular.

\section{Objectives of the Study}

The paper aims to:

1. State the prevalent educational system of the country and analyse it vis-à-vis the issue of EOs;

2. Analyse the above and briefly state the determinants of the existing situation;

3. Relate the concept of EO and education to similar situations in other countries;

4. Recommend proposals to alleviate the existing status according to the above.

\section{Limitations of the Study}

This study focuses on the primary grades, essentially because of the easy availability of data, and places the issue of EO within this level of education in Pakistan. Higher levels of education are referred to where applicable.

This paper relies on documented data that is controversial in many cases and questioned for reliability (Zia 1998). In cases where recent data is not available, older information has been utilised.

\footnotetext{
* The author has a Ph.D. in education from the University of Wales and is an Associate Professor at the College of Home Economics, Lahore.
} 
Educational sources as cited below, do not carry information on the private sector. It is accepted, though that non-government (NG) share accounts for 22 per cent of the primary sector. There is a very strong NG presence in the secondary and higher education levels, which has not been considered in the study, basically due to lack of documented data. (The researcher is aware that NG and private schools are more highly priced in general, and provide a better quality of education than the public sector. This means that children from the lower socio-economic strata lose their chance to quality education, and in later life this factor, even when considered as the sole variable, places them at a disadvantage when competing for jobs in later life).

Lack of information relating to in-school situation has meant limiting the study to enrolments and completion rates. This has meant more emphasis on out-of-school factors, rather than in-school factors.

EO concerning handicapped/exceptional children has not been discussed in the study.

\section{Significance}

Most social development studies state discrimination, bias, stereotyping, labeling, (UNICEF 1998, PIHS 1996-97) as issues that pervade various sections of the country. And yet to date, the concept of EO as a theme by itself is not mentioned in policy making or in policy implementation arenas. This study hopes to disseminate an awareness of the concept, and place the concept as a viable theme for educational settings in particular.

As a teacher in government colleges for the past 25 years, the issue of EOs has been evident in one way or the other. Involvement in social development programmes as an NGO worker has motivated the researcher to highlight the issue of EO for the social consciousness of the policy makers and planners.

\section{Methodology of the Study}

The study will rely on available published statistical data to describe the prevalent situation of the educational system. The researcher will use analytical and reflective skills to assess the situation and state possible causes for the EO situation as identified. The review of related literature will be used to identify ways of improving the above situation so that the concept of EO can be recommended as a policy for the educational system of the country. 


\section{Educational Data/Information}

The following pages will identify and delineate data that is relevant to the educational system of the country. It will focus on the primary level.

Table-1: Gross Enrollment (Proportion of children 5-11 years. Presently enrolled in Primary schools)

\begin{tabular}{cc}
\hline Pakistan & $70 \%$ \\
Urban & $78 \%$ \\
Rural & $63 \%$ \\
Boys & $74 \%$ \\
Girls & $65 \%$ \\
Provinces & \\
Punjab & $75 \%$ \\
Sindh & $70 \%$ \\
BWFP & $56 \%$ \\
\hline
\end{tabular}

Source: UNICEF (1998 p 39)

The above table clearly shows the difference in the enrolment of the primary age group ( 100 per cent enrolment is the government requirement). It also shows the difference that exists among the provinces, the rural/urban and the male/female. The same is again obvious in the following table:

Table-2: School Entry Rate: Proportion of Children of School Entry Age who are Currently Attending Primary Schools

\begin{tabular}{cc}
\hline Pakistan & $37 \%$ \\
Urban & $44 \%$ \\
Rural & $32 \%$ \\
Gender & $38 \%$ \\
Male & $36 \%$ \\
Females & \\
Provinces & $42 \%$ \\
Punjab & $36 \%$ \\
Sindh & $27 \%$ \\
NWFP & $38 \%$ \\
Balochistan & \\
\hline
\end{tabular}

Source: UNICEF (1998 p 40) 
The gross enrolment rate (GER) shows the same pattern that is visible in the above tables. The highest rate were found in the Punjab, followed by Sindh and NWFP and then Balochistan. GERs were higher in urban areas compared to rural areas (87 and 63 per cent respectively) and for boys relative to girls (78 and 61 per cent respectively). The difference between GERs for girls and boys are considerably higher in rural areas compared to urban areas (PIHS 1998 p 25).

Literacy is the most important indicator in the education sector. It also shows the urban rates to be higher than those in rural area, with wide differences in the provinces as well. The gender bias is very evident as well (Table-3).

Table-3: Literacy Population 10 years and Older---By Region and Province

\begin{tabular}{cccc}
\hline Region and Province & Percentage of Population 10 years and Older \\
& Male & Female & Both \\
\hline Urban Areas & 65 & 50 & 58 \\
Punjab & 64 & 51 & 57 \\
Sindh & 67 & 54 & 61 \\
NWFP & 58 & 34 & 46 \\
Balochistan & 61 & 27 & 45 \\
Rural Areas & 44 & 17 & 31 \\
Punjab & 45 & 21 & 32 \\
Sindh & 46 & 12 & 30 \\
NWFP & 43 & 13 & 27 \\
Balochistan & 41 & 5 & 24 \\
Overall Pakistan & 51 & 28 & 39 \\
Punjab & 51 & 30 & 40 \\
Sindh & 57 & 33 & 45 \\
NWFP & 46 & 17 & 30 \\
Balochistan & 44 & 9 & 27 \\
\hline
\end{tabular}

Source: $\quad$ PIHS: Round 2: 1996-97, p 44.

The indicators in the above data are consistent. A wide difference is evident in the educational figures for the various provinces, urban and rural areas and for boys and girls. In the light of the above it is not wrong to assume that a bias exists in the educational system and that EO, as a practice, is not a viable force in the machinations of the education system of the country. Understandably, the bias compounds with the progression in the levels of education. 
The determinants of poor education indicators are cited as quality of education, access and condition of schools, poor funding by government and poor management of the education system (UNICEF $1998 \mathrm{pp} 41-43$ ). It is also worth noting that PIHS data (1998 pp 34-35) tabulates data on 'Reasons for Never Attending School'. The stated responses were significant. The reason of "Parents did not allow" when considered as a first and second response, showed as 18 per cent of the reason for boys but in case of girls it accounted for 57 per cent of the responses. It is interesting to note that in the case of girls, 50 per cent of the cases stated demand related factors, and especially for rural girls, these factors are more important than for either boys and girls in urban areas. This establishes the fact that discrimination against females exists in the socio-cultural patterns of the country.

A positive relationship between household income and school attendance has been found in every province and region (PIHS 1996-97 pp 13-14). It can be safely assumed that the poorer the family, the less the chances for the young to attend school. This delineates the fact that socio-cultural biases are further compounded by poverty. This aggravates the discrimination and adds another dimension of 'haves' and 'have-nots' to this debate. (Due to the brevity of the report, this will not be discussed in detail).

PIHS (1998 pp 70-72) data show that 22 per cent of the primary age group children attend non-government schools. Figures state no difference in enrolments in terms of gender in this sector. But the data does show variations of greater NG enrolment in terms of urban rather than rural areas. Positive correlation was found in the income of the household and education of parents to the child attending a non-government school. Surprisingly, a high number of children from low-income groups attend NG schools and the reason is that NG schools often provide higher quality education than government schools. This provides reasonable proof that quality education is a requirement along with issues of equality and access. This is similar to conclusions reached in the West (Runnymede Trust 1993; Riley 1994).

The above data consistently show the same pattern. A wide difference is evident in the educational figures for the various provinces, urban and rural areas and then in figures for boys and girls. In the light of the above, it is not wrong to assume that a bias exists in the educational system and that EO, as a practice, does not exist for all the provinces, or the rural and urban areas or even for males and females. Provision of basic education is a state responsibility and a right of every citizen. Since it is established that lower income means higher rate of children not attending school, it becomes evident that the poor segment of the population stands 
more at risk of not getting education and the myriad benefits that accompany it in terms of better quality of living. This establishes an increased responsibility on the public sector to provide education to all across the board so that income disparity does not effect the opportunities to a better quality of life, either at home or at work.

At higher grades, the situation becomes complex. Students who have been left out of primary education because of poverty or parent's attitudes (attitude has been found to be related to parent's education and socioeconomic status (PHIS 1998 pp 40-41), or due to poor provision of infrastructure or facilities, give up their claim to higher levels of education anyway. This aggravates their claims to equality and access to equality in later life as well.

\section{Related Literature}

In the Western world EO is gaining momentum for reform, especially in the field of education. There is a call to recognise cultural diversity. Similarities rather than differences within the various cultures are being stressed. The multicultural approach is being increasingly encouraged in a wider context in general, and educational settings in particular (Antonouris and Wilson 1989 pp 5-6). The talk is about a "democratic pluralist society" (Swann Report 1995). Teaching-Learning environment is placed within the perspective of EO. Teachers are being increasingly entrusted with the task of creating awareness of the concept, thus helping undermine stereotyping, bias and discriminatory behaviour (Pratt et al 1984, Blackstone 1985 p xiii, Antonouris and Wilson 1989 pp 101-102, Orr 1995 p 11 and 18). Teaching materials reflect the same approach. This has led to the evolution of the concept of Education for All (EFA). The idea is to educate all with a focus on shared values and appreciation of the diversity of culture, religion, language, race, gender and so on. Most developed countries of the world anticipating the possible friction arising in their societies due to the vast diversity of their nationals have initiated the concept of EO in their curriculum, teaching objectives, classroom environment, instructional materials and teaching practice. Devising a curriculum that will reflect the above and focus on ideas, principles and generalisations for EO concepts in particular are the main test for an expert. Devising such a curriculum is the need of the time.

\section{Conclusions and Recommendations}

The EO concept in its totality is a recent development all over the world. Its major components, that is, bias, discrimination, inequality have long held negative connotations in general and for education in particular. 
Being an Islamic ideological state, Pakistan has propounded "no difference on basis of class, creed, colour, race, social class and so on", and yet the state has to honour its commitment in practice in general, and education in particular.

The above literature clearly pinpoints the vast difference in perspectives of developed countries and Pakistan, an underdeveloped economy. Both are tackling the issue of EO, but at different levels. Where Pakistan has yet to initiate a socio-cultural attitudinal change in the populace, the West is tackling the issue within its classrooms. The West has the added advantage of 100 per cent enrolments in its schools, which is not so in the case of Pakistan.

Pakistan clearly needs to tackle the issue of EO at the community leve1. Its first challenge would be to fulfill the quantity requirements for education. This is not to say that quality issues cannot be tackled alongside. There is a need for initiative for EO practices both inside and outside the schools and both need to be addressed concurrently.

All educational policies, time and again have proposed equality. Most social development policies, such as the National Plan of Action for Women Development are tackling the issues of gender bias and discrimination. This study propounds that the whole range of issues encompassed in EO, of which gender bias is one, be dealt with on a common platform. This would mean a more effective and sustainable solution to the existing situation in the country where there is a lack of EO that is area-based, gender-based, social class based, to name a few. 


\section{References}

Antonouris, G. and Wilson, J., 1989, Equal Opportunities in Schools: New Dimensions in Topic Work. New York: Casse11/Nichols Publishing.

Blackstone, T., 1985, Preface. Whyte et al (Eds.) Girl Friendly Schooling. London: Routledge.

Clements, P. and Spinks, T., 1994, The Equal Opportunities Guide: How to deal with issues of everyday unfairness. London: Kogan Page.

Orr, P., 1985, Sex bias in schools: national perspectives. Whyte et al (Eds.) Girl Friendly Schooling. London: Routledge.

Pakistan Integrated Household Survey. (n.d.). PIHS. Round 2. 1996-97. Islamabad: Federal Bureau of Statistics, Government of Pakistan.

Pakistan Integrated Household Survey, 1998, PIHS. Education Sector Performance in the 1990s. Islamabad: Federal Bureau of Statistics, Government of Pakistan.

Pratt, J.; Bloomfield, J. and Seale, C., 1984, Option Choice. A Question of Equal Opportunity. Slough: NFER-Nelson.

Pratt, J., 1985, The attitudes of teachers. Whyte et al (Eds.) Girl Friendly Schooling. London: Routledge.

Runnymede Trust, 1993, Education For All. London: HMSO.

UNICEF., 1998, 'The Situation Analysis of the Woman and Child in akistan', Islamabad: UNICEF.

Zia, R., 1998, Profile of the Rural Woman of Pakistan, In The Lahore Journal of Economics, 3,1, 47-79. 